\title{
Evolution of postcranial skeleton in worm lizards inferred from its status in the Cretaceous stem-amphisbaenian Slavoia darevskii
}

\author{
Mateusz Tałanda \\ Acta Palaeontologica Polonica 62 (1), 2017: 9-23 doi:https://doi.org/10.4202/app.00294.2016
}

Amphisbaenians are one of the most derived fossorial groups among Squamata. Studies of their evolution were hampered by their specialized limbless morphology and unrecognized early fossil record. This study presents a detailed description of the postcranial anatomy of the oldest known stem-amphisbaenian Slavoia darevskii. The skeleton shows an incipient adaptation to the fossorial mode of life, expressed in the early stages of limb reduction and elongation of the trunk, typical aspects of modern worm lizards. The forelimbs show a hyperphalangy of the first digit. They were probably strengthened this way to support the head in burrowing. Such an anatomy of the most ancient amphisbaenians implies that the forelimbs were lost multiple times in the amphisbaenians to be retained only in the Bipedidae. The hindlimb reduction was initiated early (before the split into modern families) but may have been completed independently. Despite these changes, $S$. darevskii still had the plesiomorphic 26 presacral vertebrae but the neck region was shorter, as shown by the ribs morphology and position.

Key words: Amphisbaenia, limb loss, hyperphalangy, parallel evolution, Cretaceous.

Mateusz Tałanda [m.talanda@biol.uw.edu.pl], Department of Paleobiology and Evolution, Faculty of Biology, Biological and Chemical Research Centre, University of Warsaw, ul. Żwirki i Wigury 101, 02-089 Warszawa, Poland.

This is an open-access article distributed under the terms of the Creative Commons Attribution License (for details please see creativecommons.org), which permits unrestricted use, distribution, and reproduction in any medium, provided the original author and source are credited. 
FaF Full text $(1,016.8 \mathrm{kB})$

For ${ }^{2}$ - Supplementary file $(991.3 \mathrm{kB})$ 